\title{
Liberation of eicosapentaenoic acid and degradation of the major cell wall polysaccharide porphyran by fermentation of nori, the dried thalli of Pyropia yezoensis, with koji
}

\author{
Yoichiro Hama $^{1,2}$ (D) Emi Yamagata ${ }^{1} \cdot$ Noriko Takahama $^{1} \cdot$ Yuka Yoshimura $^{1} \cdot$ Rin Yanagida $^{1} \cdot$ Susumu Mitsutake $^{1,2}$
}

Received: 24 June 2021 / Revised: 5 August 2021 / Accepted: 5 August 2021 / Published online: 8 October 2021

(c) The Author(s) 2021

\begin{abstract}
In this study, dried sheets of nori, shredded and processed thalli of the red alga Pyropia yezoensis, were fermented with either barley, rice or soybean koji. High-performance liquid chromatographic analyses of the lipid extracts of the fermented products indicated that the fermentation of nori with all kinds of tested koji released free fatty acids, including the eicosapentaenoic acid, from ester lipids. We found that approximately half of the eicosapentaenoic acid in nori had been released as the free fatty acid at up to 4 weeks of fermentation at $30{ }^{\circ} \mathrm{C}$ and more than $65 \%$ at 8 to 12 weeks in the fermented products with barley and rice koji. We also demonstrated the degradation of porphyran, a major cell wall polysaccharide of nori, by gel chromatography on Sephacryl S-300 HR of hot water extracts of the fermented products of nori with barley koji. Approximately two-third of porphyran had been degraded to porphyran oligosaccharides up to 6 weeks of fermentation. Fermentation of nori with koji may bring out the potential health-promoting functions of nori.
\end{abstract}

Keywords Eicosapentaenoic acid $\cdot$ Porphyran $\cdot$ Pyropia yezoensis $\cdot$ Koji $\cdot$ Fermentation $\cdot$ Nori

\section{Introduction}

Nori (laver) is an ingredient used in sushi and other dishes, and with the popularity of Japanese food, it is widely consumed not only in Japan and other East Asian countries but also throughout the world. In Japan, the raw material for most nori is the cultivated thalli of Pyropia yezoensis. After being shredded and dried, the thalli are often processed into dried sheets. Red algae, such as Pyropia, are known to contain eicosapentaenoic acid (EPA), most of which is a constituent of glycoglycerolipids in chloroplasts (Harwood 1998; Kumari et al. 2013). The fatty acid composition of Pyropia thalli is unique: EPA accounts for about half of the total fatty acids with no detectable docosahexaenoic acid (Araki et al. 1986; Noda 1993). A dried sheet of nori contains $3.7 \%$ crude

Yoichiro Hama

hamay@cc.saga-u.ac.jp

1 Applied Biochemistry and Food Science Course, Faculty of Agriculture, Saga University, 1 Honjo, Saga 840-8502, Japan

2 The United Graduate School of Agricultural Sciences Kagoshima University, Kagoshima, Japan lipid (MEXT Japan 2015), suggesting that its EPA content is almost $2 \%$. Since the human intake of $n-3$ polyunsaturated fatty acids (PUFA), such as EPA, has been indicated to be insufficient for preventing lifestyle-related diseases and metabolic syndrome, algae are attracting attention as alternative sources of n-3 PUFA (Swanson et al. 2012; Kumari et al. 2013; Wells et al. 2017). However, its effects on biological processes, such as digestion and the bioavailability of nutrients from algae, are still not well understood (Wells et al. 2017). One of the major reasons for this is that algae are rich in viscous and indigestible cell wall polysaccharides, such as porphyran, xylan, and mannan, found in Pyropia (Painter 1983).

Koji is produced by multiplying grains such as rice, barley, and soybeans with microorganisms, such as Aspergillus oryzae. Enzymes produced by microorganisms in koji play major roles in the production of fermented Japanese traditional foods, such as sake, miso, and soy sauce (Hesseltine 1983; Zhu and Tramper 2013). These enzymes include starch-hydrolyzing, proteolytic, and lipolytic enzymes (Hesseltine 1983; Ohnishi et al. 1994; Zhu and Tramper 2013). The action of enzymes on koji changes the food components during fermentation, resulting in the unique characteristics of individual fermented foods. 
In this study, we focused on EPA and porphyran, a major cell wall polysaccharide of nori, and explored the changes in these components during fermentation of nori with koji.

\section{Materials and methods}

\section{Reagents and materials}

The reagents used in this study were special grade products from Fujifilm Wako Chemicals (Japan), unless otherwise noted. Acetonitrile and methanol $(\mathrm{MeOH})$ used for high-performance liquid chromatography (HPLC) were of HPLC grade (Fujifilm Wako Chemicals). The ADAM reagent (9-anthryldiazomethane, fluorescent reagents for fatty acid analysis) was purchased from Funakoshi (Tokyo, Japan). Dried sheets of nori made from thalli of Pyropia yezoensis, which had been cultivated in the Ariake Sea, were purchased from a local market in the Saga prefecture, Japan. Barley, rice, and soybean koji were obtained commercially (Suzuki Koji Store, Shizuoka, Japan).

\section{Fatty acid composition of nori}

The fatty acid composition of the nori sample used in this study was determined using gas-liquid chromatography (GLC). Crude lipids were prepared from $1.0 \mathrm{~g}$ of the nori sample using the method described by Bligh and Dyer (1959). Fatty acid methyl esters were prepared by transesterification of crude lipids with methanolic hydrogen chloride $(\mathrm{HCl})$ (hydrogen chloride-methanol reagent (5-10\%) for esterification; Tokyo Chemical Industry, Japan) (Christie, 1973). The esters thus obtained were extracted with hexane, concentrated, and analyzed using GLC. GLC analysis was performed on a Shimadzu GC-2010 instrument (Shimadzu, Japan) equipped with a capillary column (BPX90, $0.25 \mu \mathrm{m}$ thickness, $0.25 \mathrm{~mm}$ i.d. $\times 30 \mathrm{~m}$; SGE Analytical Science, Australia). Methyl nonadecanoate (99\%; GL Sciences, Japan) was used as the internal standard for quantification.

\section{Preparation of fermented products of nori with koji}

Ten sheets of nori ( $30 \mathrm{~g})$ were cut into small pieces using scissors. Then, salt (10 g, $\geq 99 \%$ sodium chloride; The Salt Industry Center of Japan, Japan), barley, rice, or soybean $k o j i(30 \mathrm{~g})$ and distilled water $(30 \mathrm{~mL})$ were added. The mixture was ground and mixed using a food processor. Each of the preparations was transferred into an airtight container and kept at $30{ }^{\circ} \mathrm{C}$ with stirring twice a week.

\section{Extraction of crude lipids}

Crude lipids were extracted according to the procedure described by Bligh and Dyer (1959). Briefly, to the fermented products $(5.0 \mathrm{~g})$, chloroform $\left(\mathrm{CHCl}_{3}\right)(5.5 \mathrm{~mL})$, $\mathrm{MeOH}(11.0 \mathrm{~mL})$, and distilled water $(2.4 \mathrm{~mL})$ were added. The mixture was shaken vigorously and centrifuged at $3000 \mathrm{rpm}$ for $10 \mathrm{~min}$. The resultant supernatant was transferred to another tube, and the extraction from the precipitate was repeated. To the combined supernatant, $\mathrm{CHCl}_{3}$ $(11.0 \mathrm{~mL})$ and distilled water $(11.0 \mathrm{~mL})$ were added. The solution was shaken and kept at $5{ }^{\circ} \mathrm{C}$ overnight and the lower lipophilic layer was separated and evaporated in vacuo.

\section{Preparation of the free fatty acid fraction}

Free fatty acids in the crude lipids were separated by solidphase extraction using a Mega Bond Elut- $\mathrm{NH}_{2}$ cartridge (500 mg, $6 \mathrm{~mL}$; Agilent, USA) according to Bernhardt et al. (1996). Briefly, $400 \mu \mathrm{L}$ aliquots of crude lipids dissolved in $\mathrm{CHCl}_{3}(4 \mathrm{~mL})$ were applied to a cartridge that had been conditioned with hexane $(2 \mathrm{~mL})$. Phospholipids and neutral lipids, except for free fatty acids, were eluted with $\mathrm{CHCl}_{3}$ :2-propanol $(2 / 1, \mathrm{v} / \mathrm{v})(4 \mathrm{~mL})$, and then, free fatty acids were eluted with $2 \%$ acetic acid in diethyl ether $(4 \mathrm{~mL})$. The eluate was then made up to $5 \mathrm{~mL}$ with diethyl ether to obtain the free fatty acid fraction.

\section{Preparation and determination of fatty acid fluorescent derivatives}

Fluorescent derivatives of free fatty acids were prepared using ADAM reagent according to Nimura and Kinoshita (1980). The fatty acid fraction $(200 \mu \mathrm{L})$ was mixed with $4.0 \mu \mathrm{g}$ of decanoic acid (from pain, $\geq 99 \%$ ) as the internal standard. The sample was concentrated using a stream of nitrogen gas. To the dried sample, $200 \mu \mathrm{L}$ of the ADAM reagent was added. Then, it was kept for $1 \mathrm{~h}$ at room temperature in the dark. The derivatized sample was stored at $-40{ }^{\circ} \mathrm{C}$ until subsequent HPLC analysis. Fatty acid fluorescent derivatives were separated by HPLC according to the method described by Suzuki (1994). Briefly, a Cosmosil 5C18-P column $(4.6 \times 250 \mathrm{~mm}$; Nacalai Tesque, Japan) with a guard column (Cosmosil 5C18-ARII, 4.6×10 mm) was connected to an HPLC pump (PU-2089; Jasco, Japan). After injection of the sample, it was eluted with acetonitrile: $\mathrm{MeOH}: \mathrm{H}_{2} \mathrm{O}(8 / 1 / 1$, $\mathrm{v} / \mathrm{v} / \mathrm{v}$ ) (solvent $\mathrm{A}$ ) and $99.9 \% \mathrm{MeOH}$ (solvent $\mathrm{B}$ ) at a flow rate of $1.1 \mathrm{~mL} / \mathrm{min}$. The solvent system used for the elution was as follows: 0-15 min, $100 \%$ solvent A; $15-55$ min, a linear gradient of $100 \%$ A to $100 \% \mathrm{~B} ; 55-90 \mathrm{~min}, 100 \% \mathrm{~B}$. The fatty acid fluorescent derivatives in the eluant were detected using a fluorescence detector (FP-920; Jasco) with an excitation maximum at $365 \mathrm{~nm}$ 
and emission maximum at $412 \mathrm{~nm}$. To prepare a standard curve for the determination of EPA, known amounts of authentic EPA ( $\geq 99 \%$; Sigma-Aldrich, USA) were mixed with decanoic acid $(4.0 \mu \mathrm{g})$. The sample was derivatized and analyzed using HPLC, as described above.

\section{Extraction and fractionation of porphyran and porphyran oligosaccharides}

To the fermented products $(1.0 \mathrm{~g}), 100 \mathrm{~mL}$ of distilled water was added. The mixture was heated at $100{ }^{\circ} \mathrm{C}$ for $90 \mathrm{~min}$ with occasional shaking to extract porphyran and porphyran oligosaccharides (oligo-porphyran). The mixture was then centrifuged at $8000 \mathrm{rpm}$ for $10 \mathrm{~min}$. Three milliliter of the clear supernatant was applied to a Sephacryl S-300 HR gel filtration column $(2.6 \times 100 \mathrm{~cm}$; Sigma-Aldrich) with $0.2 \mathrm{M}$ sodium chloride containing $0.1 \%$ n-butanol as the eluent. Elution of porphyran and oligo-porphyran in the eluant was monitored by detecting anhydrogalactose, a constituent monosaccharide of porphyran and oligo-porphyran, with resorcinol reagent as described by Yaphe and Arsenault (1965). 3,6-Anhydro-D-galactose ( $\geq 98 \%$; SigmaAldrich) was used as the reference compound for quantification. The molecular weights of porphyran and oligo-porphyran in the eluate were determined from the elution positions of pullulan molecular weight markers (Shodex, Japan).

\section{Solubility of EPA in the fermented products}

The solubility of EPA in the fermented products in the external aqueous solution was determined. First, $0.1 \mathrm{M}$ sodium bicarbonate $(10 \mathrm{~mL})$ with or without $2 \mathrm{mM}$ sodium bile acid was added to the fermented products $(0.5 \mathrm{~g})$. The mixture was then heated at $37^{\circ} \mathrm{C}$ for $10 \mathrm{~min}$ with shaking. Then, it was centrifuged at $3000 \mathrm{rpm}$ for $10 \mathrm{~min}$ to obtain a clear supernatant. The supernatant was transferred to another test tube. After the addition of two drops of concentrated acetic acid to make the solution acidic, substances including free fatty acids and other lipophilic compounds dissolved in the supernatant were extracted with diethyl ether $(5.0 \mathrm{~mL})$. The ether solution was concentrated using a stream of nitrogen gas. In order to quantify not only the free EPA but also the ester form of EPA, fatty acid methyl esters were prepared by transesterification of the extracts with methanolic $\mathrm{HCl}$ and analyzed by GLC, as described above.

\section{Results}

\section{Liberation of free EPA}

The fatty acid composition of the dried sheets of nori used in this study was determined. The major fatty acids were EPA and hexadecanoic acid, and almost $80 \%$ were occupied by these two fatty acids. Contents in $1.0 \mathrm{~g}$ of the nori sample and percentages in total fatty acids were as follows: EPA, $21.9 \mathrm{mg}$ (54.2\% of total fatty acid); hexadecanoic acid, $9.3 \mathrm{mg}$ (24.0\% of total fatty acid). The results agreed with those already reported (Noda 1993) and showed that $10 \mathrm{~g}$ of the preparation for fermentation contained $65.7 \mathrm{mg}$ of EPA. The free EPA ratio in the fermented products described below was calculated based on this value. Since EPA was not detected in the fermentation process of soybean and barley koji (Feng et al. 2014), it is obvious that the EPA detected in this study originated exclusively from nori.

As the fermentation period progressed, the salt and koji grains in the fermented products became smaller, the viscosity became stronger, and the glaze came out. After extraction of crude lipids from the fermented products and subsequent preparation of the free fatty acid fraction, free EPA was separated and quantified as its fluorescent derivative by

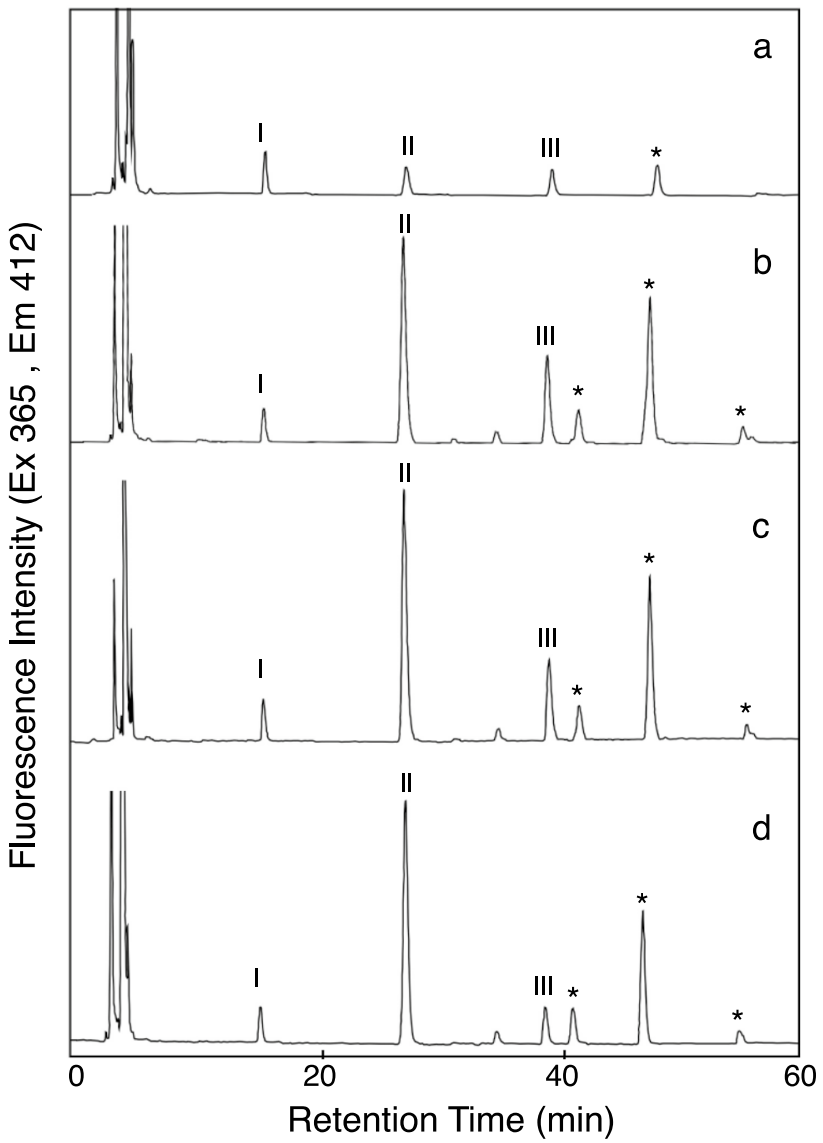

Fig. 1 Separation and determination of free eicosapentaenoic acid (EPA) in the fermented products of nori with the barley koji. The fluorescence derivatives of free fatty acids were separated and analyzed by high-performance liquid chromatography on Cosmosil 5C18-P. The crude lipids were extracted from the fermented products with barley koji at $30{ }^{\circ} \mathrm{C}$ for 0 weeks (before fermentation) (a), 3 weeks (b), 6 weeks (c), and 12 weeks (d), respectively. Peaks I, decanoic acid (internal standard); II, EPA; III, hexadecanoic acid. As per the elution positions and fatty acid compositions of nori, the peaks marked with asterisks were not fatty acid derivatives originating from nori. Detailed conditions are described in the text 
HPLC (Fig. 1). Free EPA (peak II in Fig. 1) and free hexadecanoic acid (peak III) were detected. The ratio of free EPA to total EPA gradually increased with fermentation time, showing a maximum at 8- to 12-week fermentation, with both barley (Fig. 2a, b) and rice koji (Fig. 2c), and then decreased. A similar liberation profile of hexadecanoic acid, another major fatty acid component in nori, during the fermentation process was observed. To verify the changes in the ratio of EPA during fermentation with barley koji (Fig. 2a), another fermentation test with barley koji under the same conditions was conducted (Fig. 2b). Considering the results of these experiments, as well as those of the experiments with rice koji (Fig. 2c), it was confirmed that the release of EPA during the fermentation period proceeded as shown in the curves depicted in Fig. 2. Surprisingly, the maximum release ratio of EPA reached more than $65 \%$ in the fermented products with both barley and rice koji (Fig. 2a-c). The decrease in the free EPA ratio at the final stage of the fermentation period, that is, 10- to 14-week fermentation, was thought to be due to the higher consumption rate of free EPA by the koji and coexisting microorganisms than the liberation rate of it from the ester lipids. For the fermented products with soybean koji, although similar EPA release could also be confirmed at the initial stage of the fermentation period, mold development, which covered the surface of the fermented products, was apparent during the 4-week fermentation period. Therefore, fermentation tests with soybean koji ended there. The reason for the mold growth was considered to be that soybeans are richer in nutrients, so they are more susceptible to mold growth than others.

\section{Degradation of porphyran}

To examine the degradation of a major cell wall polysaccharide, porphyran, during the fermentation of nori with koji, hot water extracts were prepared using fermented products with barley koji after 0,6 , and 12 weeks of fermentation. Porphyran is mainly composed of D-galactose and either L-galactose-6-sulfate or anhydro-L-galactose (L-AG) (Rees and Conway 1962), and AG is distributed only in some red algae such as Pyropia (Yalpani 1988). Therefore, porphyran and oligo-porphyran in the eluate of gel filtration can be monitored specifically by detecting AG with no interference from other hot water-soluble carbohydrate components such as starch from grains and floridosides from nori (Noda et al. 1981). When the extract from the preparation before fermentation ( 0 -week fermentation period) was fractionated by gel filtration on Sephacryl S-300 HR, only one peak of
Fig. 2 Changes in the free EPA ratios during the fermentation of nori with barley, rice, and soybean koji at $30^{\circ} \mathrm{C}$. A dried sheet of nori was fermented with barley koji (a), barley koji (another preparation) (b), rice $k o j i(\mathbf{c})$, and soybean koji (d), respectively. To confirm the changes in the free EPA ratios during the fermentation with barley koji (a), another fermentation test (b) was conducted under the same conditions. For the fermented products with soybean koji (d), mold development, which covered the surface of the fermented products, was apparent during the 4-week fermentation period. Therefore, fermentation tests with soybean koji ended there. Detailed conditions are described in the text
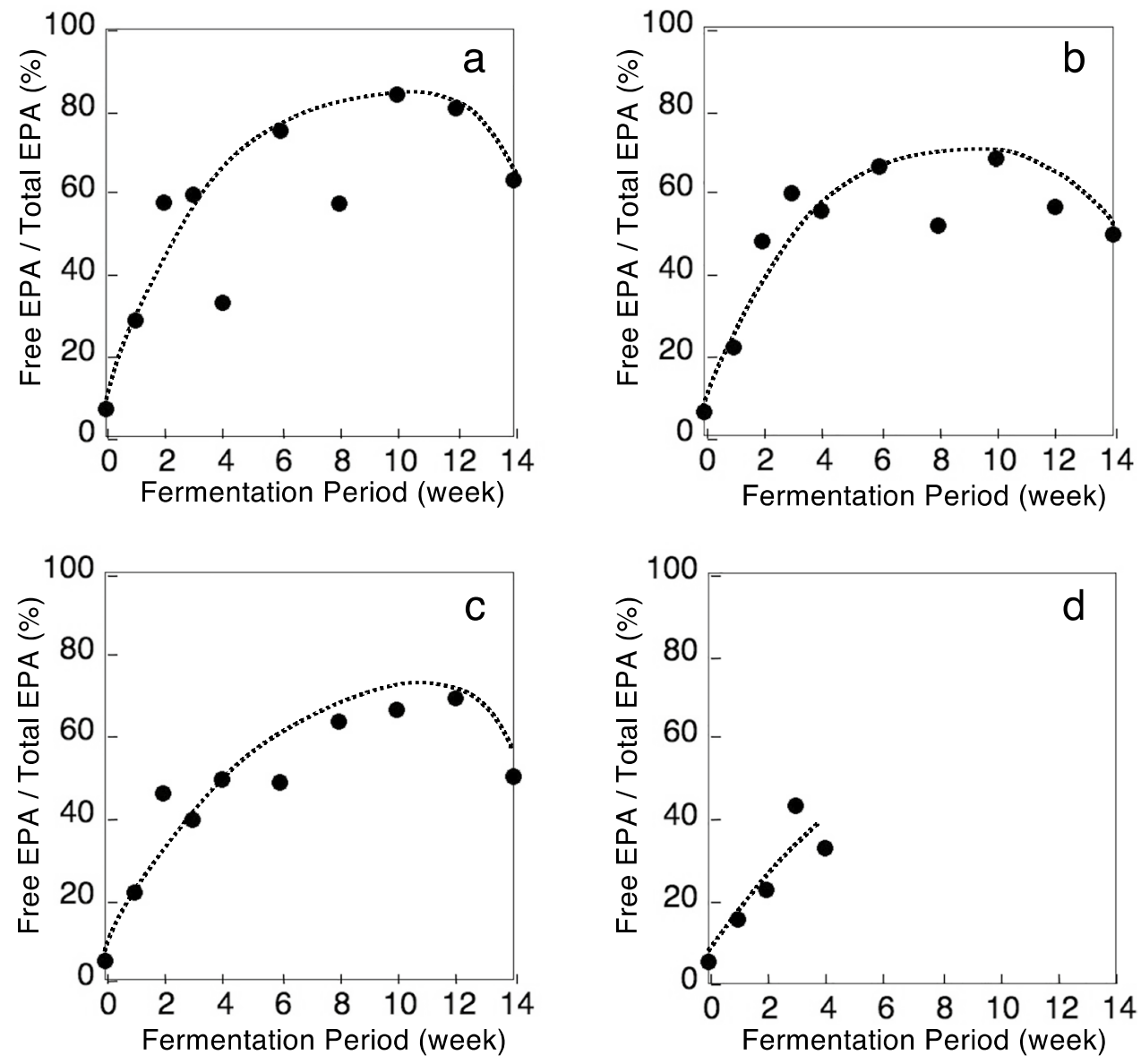
AG-containing saccharides was detected at Fr. No. 37-49 (Fr. I in Fig. 3a). Deduced from the elution positions of pullulan molecular weight markers, the molecular weight of Fr. I, which could be assigned to be porphyran originally present in nori, was determined to be $1.9 \times 10^{5}$ to $4.8 \times 10^{5}$. No degradation products of porphyran were detected after the elution of porphyran (Fr. I). On the other hand, in the extracts from preparations after 6 and 12 weeks of fermentation, native porphyran eluted at Fr. I decreased with a concomitant increase in the degradation products of porphyran and oligo-porphyran (Frs. II and III in Fig. 3b, c), as detected widely after elution of Fr. I. In the hot water extract after 6 weeks of fermentation, distribution of AG-containing saccharides, namely, porphyran and oligo-porphyran, was 32.5 (Fr. I), 20.6 (Fr. II), and 46.9\% (Fr. III), respectively.
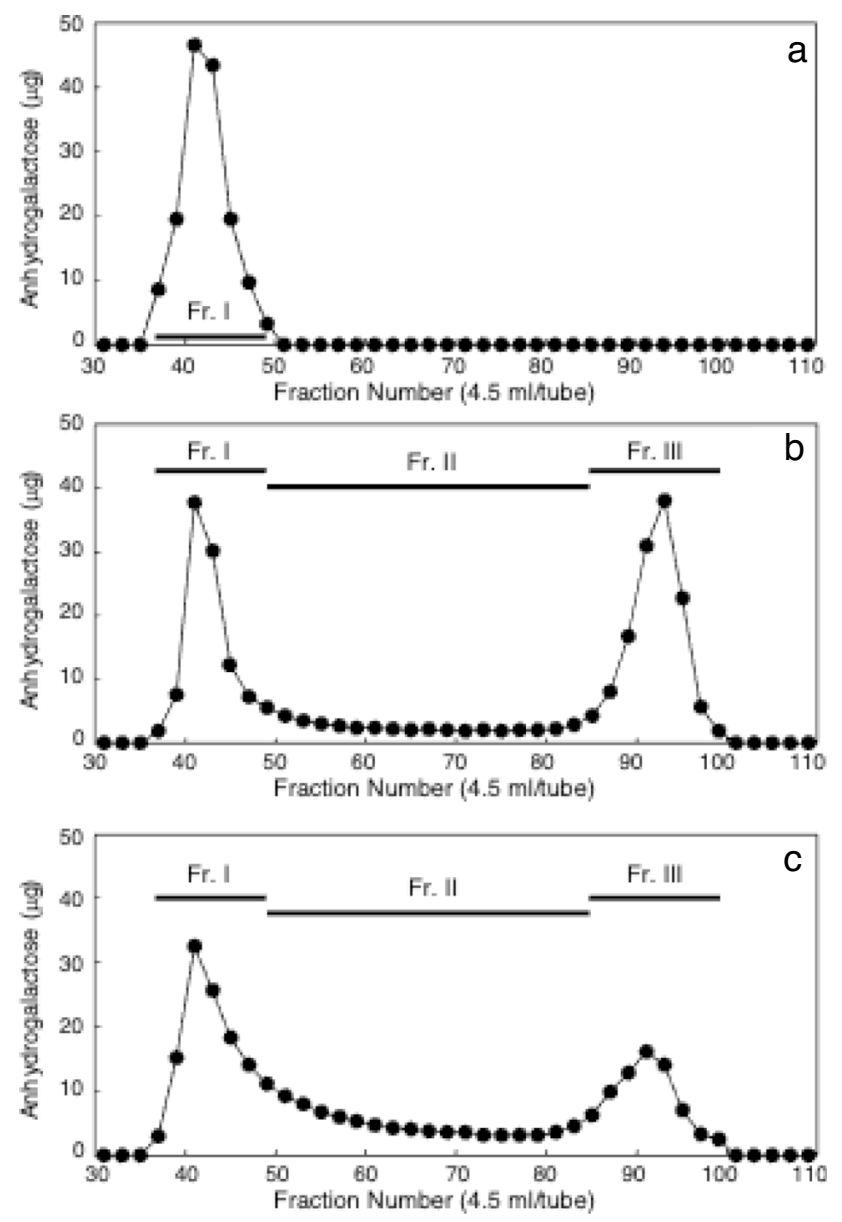

Fig. 3 Gel filtration of porphyran and porphyran oligosaccharides from the fermented products with barley koji on Sephacryl S-300 HR. Porphyran and porphyran oligosaccharides were extracted with hot water from the fermented products with barley koji at $30{ }^{\circ} \mathrm{C}$ for 0 weeks (before fermentation) (a), 6 weeks (b), and 12 weeks (c), respectively. The elution of porphyran and porphyran oligosaccharides was monitored by detecting the anhydrogalactose in the eluate using the resorcinol reagent. Column size, $2.6 \times 100 \mathrm{~cm}$; eluent, $0.2 \mathrm{M}$ sodium chloride containing $0.1 \% \mathrm{n}$-butanol. Detailed conditions are described in the text
Molecular weights of oligo-porphyrans in Fr. II (Fr. No. 50-85), and Fr. III (Fr. No. 86-103) were determined to be $1.9 \times 10^{5}$ to $2.3 \times 10^{3}$ and less than $2.3 \times 10^{3}$, respectively. Since the fractionation range of the Sephacryl S-300 HR gel is 1 to $400 \mathrm{kDa}$ for dextran (Merck KGaA 2021), oligoporphyrans with molecular weights less than $1 \mathrm{kDa}$, which are equivalent to oligosaccharides with a degree of polymerization of less than 5 or 6 , were considered to be eluted together at Fr. III without separation, resulting in one peak formation at Fr. III. Oligo-porphyrans eluted at Fr. II, which were partially degraded porphyran, tended to accumulate with increasing fermentation period. In contrast, oligo-porphyran at Fr. III accumulated once and then decreased as the fermentation period progressed. The lower molecular weight oligosaccharides in Fr. III are thought to be readily assimilated by coexisting microorganisms.

\section{Solubility of EPA in the fermented products}

Before fermentation of nori with barley koji, no EPA was detected in aqueous sodium bicarbonate as the solvent (Table 1). Even in solvents containing bile acid, a biosurfactant that acts in the human intestine, little EPA was detected. Therefore, it was suggested that most of the lipids, including EPA originally present in nori, will be excreted without being digested, absorbed, or utilized. In contrast, EPA in the 11-week fermented products was found to be solubilized in both solvents with and without bile acid. Therefore, by fermentation of nori with koji, it was suggested that at least a part of the EPA was useful for humans.

\section{Discussion}

Koji was used as the starter for fermentation in this study, as in other fermented Japanese traditional foods, such as sake, miso, and soy sauce. Koji is prepared by inoculating fungi such as Aspergillus oryzae to grains, but other microorganisms originally present in koji, such as yeast and bacteria, are also the important benefactors for the fermentation with $k o j i$. During fermentation process of these foods in presence of koji, contamination from environmental microorganisms will occur, but the contaminants cannot overcome the normal flora present in koji (Hesseltine 1983). Therefore, it can be deduced that changes in nori components found in this study are attributable predominantly to the actions of enzymes derived from microorganisms originally present in koji.

Fatty acids and fatty acid ethyl esters are produced in fermented foods made from koji, such as miso and soy sauce, and fatty acid ethyl esters are involved in the specific aroma formation of individual fermented foods (Hesseltine 1983). The amount of EPA ethyl ester produced in the fermented 
Table 1 Solubility of eicosapentaenoic acid (EPA) in the fermented products of nori with the barley koji

\begin{tabular}{ccc}
\hline $\begin{array}{c}\text { Fermentation } \\
\text { period (week) }\end{array}$ & Solvent & $\begin{array}{c}\text { Soluble EPA } \\
\text { (mg in 10 g of ferments) }\end{array}$ \\
\hline 0 & $0.1 \mathrm{M} \mathrm{NaHCO}_{3}$ & n.d. \\
& $0.1 \mathrm{M} \mathrm{NaHCO}_{3}$ & 1.1 \\
& $+2 \mathrm{mM} \mathrm{bile} \mathrm{acid}$ & 9.2 \\
11 & $0.1 \mathrm{M} \mathrm{NaHCO}_{3}$ & 10.0 \\
& $0.1 \mathrm{M} \mathrm{NaHCO}_{3}$ & \\
& $+2 \mathrm{mM} \mathrm{bile} \mathrm{acid}$ & \\
\end{tabular}

To the fermented products $(0.5 \mathrm{~g}), 0.1 \mathrm{M}$ sodium bicarbonate $\left(\mathrm{NaHCO}_{3}\right)(10 \mathrm{ml})$ with or without $2 \mathrm{mM}$ sodium bile acid was added. The mixture was then heated at $37{ }^{\circ} \mathrm{C}$ for 10 min with shaking. After centrifugation, the EPA content dissolved in the supernatant was determined. Detailed conditions are described in the text.

products prepared in this study was less than $0.5 \%$ of the total EPA (data not shown).

An attempt to create a nori sauce by fermenting nori with koji has been reported (Uchida et al. 2017, 2018), but changes in the lipid components including EPA and cell wall polysaccharides have not been investigated yet. In this study, by the fermentation of nori with koji, it was revealed for the first time that most of the total EPA was liberated to give free EPA and that the large part of porphyran, a major cell wall polysaccharide of nori, was degraded into lower molecular weight oligosaccharides. Probably due to the free EPA formation and degradation of porphyran, EPA in the fermented products was shown to be soluble in external aqueous solvents. Porphyranase, an enzyme capable of hydrolyzing porphyran, was reported to be horizontally transferred in Japanese intestinal bacteria (Hehemann et al. 2010), so it is expected that a part of porphyran of nori might be degraded in the intestines of Japanese people.

Among PUFAs, EPA is known to be one of the most potent functional lipids, and n-3 PUFAs such as EPA and docosahexaenoic acid (DHA) have been used not only as a health food but also as a drug for hyperlipidemia. After digestion and absorption of dietary EPA and DHA, a large family of bioactive mediators such as eicosanoids and resolvins are biosynthesized, and most of the physiological functions of these n-3 PUFAs can be attributed to the functions of these potent chemical mediators (Serhan et al. 2008; Swanson et al. 2012). On the other hand, it has also been reported that free n-3 PUFAs such as EPA, DHA, and $\alpha$-linolenic acid in the diet act as ligands for receptors of enteroendocrine L-cells on the surface of the gastrointestinal tract before being digested and absorbed, thereby preventing lifestyle-related diseases such as anti-hypertension and antiobesity via induction of gastrointestinal hormone secretion (Bhaswant et al. 2015). Namely, not only the content but also the form of existence of n-3 PUFA are very important, and the presence of n-3 PUFA in free form can provide additional important value to the foods.
In conclusion, fermentation of nori with koji will bring out the potential health-promoting functions of nori, which may lead to its widespread use as a health food. Moreover, fermentation in the presence of koji, as shown in this study, is a very simple and inexpensive process for the food industry that does not require any special equipment nor expensive additives. However, by introducing this process, the valorization of seaweed, especially red algae, will be improved since the fermented seaweed products are expected to have beneficial effects on human health.

In addition, it is noteworthy that the fermented products prepared in this study were very tasty and could be used as miso.

Author contribution Yoichiro Hama: supervision, conceptualization, investigation, validation, writing — original draft, writing — review and editing. Emi Yamagata: investigation, validation. Noriko Takahama: investigation, validation. Yuka Yoshimura: investigation, validation. Rin Yanagida: investigation, validation. Susumu Mitsutake: supervision, conceptualization, writing-review and editing.

Funding This work was partially supported by Jisshinkai at the Kagoshima Prefecture, Japan.

Data availability The data that support the findings of this study are available from the corresponding author, $\mathrm{YH}$, upon reasonable request.

\section{Declarations}

Competing interests The authors declare no competing interests.

Open Access This article is licensed under a Creative Commons Attribution 4.0 International License, which permits use, sharing, adaptation, distribution and reproduction in any medium or format, as long as you give appropriate credit to the original author(s) and the source, provide a link to the Creative Commons licence, and indicate if changes were made. The images or other third party material in this article are included in the article's Creative Commons licence, unless indicated otherwise in a credit line to the material. If material is not included in the article's Creative Commons licence and your intended use is not permitted by statutory regulation or exceeds the permitted use, you will need to obtain permission directly from the copyright holder. To view a copy of this licence, visit http://creativecommons. org/licenses/by/4.0/.

\section{References}

Araki S, Sakurai T, Omata T, Kawaguchi A, Murata N (1986) Lipid and fatty acid composition in the red alga, Porphyra yezoensis. Jap J Phycol 34:94-100

Bernhardt TG, Cannistraro PA, Bird DA, Doyle KM, Laposata M (1996) Purification of fatty acid ethyl esters by solid-phase extraction and high-performance liquid chromatography. J Chromatogr B 675:189-196

Bhaswant M, Poudyal H, Brown L (2015) Mechanisms of enhanced insulin secretion and sensitivity with n-3 unsaturated fatty acids. J Nutr Biochem 26:571-584

Bligh EG, Dyer WJ (1959) A rapid method of total lipid extraction and purification. Can J Biochem Physiol 37:911-917 
Christie WW (1973) Acid-catalyzed esterification and transesterification. Lipid analysis. Pergamon Press, New York, pp 88-89

Feng Y, Chen Z, Liu N, Zhao H, Cui C, Zhao M (2014) Changes in fatty acid composition and lipid profile during koji fermentation and their relationships with soy sauce flavour. Food Chem 158:438-444

Harwood JL (1998) Membrane lipids in algae. In: Siegenthaler P-A, Murata N (eds) Lipids in photosynthesis: structure, function and genetics. Kluwer Academic Publishers, Dordrecht, pp 53-64

Hehemann J-H, Correc G, Barbeyron T, Helbert W, Czjzek M, Michel G (2010) Transfer of carbohydrate-active enzymes from marine bacteria to Japanese gut microbiota. Nature 464:908-912

Hesseltine CW (1983) Microbiology of oriental fermented foods. Annu Rev Microbiol 37:575-601

Kumari P, Kumar M, Reddy C, Jha B (2013) Algal lipids, fatty acids and sterols. In: Domíngues H (ed) Functional ingredients from algae for foods and nutraceuticals. Woodhead Publishing, Oxford, pp 87-134

Merck KGaA (2021) Sephacryl S300-HR. https://www.sigmaaldri $\mathrm{ch} . \mathrm{com} / \mathrm{catalog} / \mathrm{product} / \mathrm{sigma} / \mathrm{s} 300 \mathrm{hr}$ ?lang=en\& region $=$ US . Accessed 7 June 2021

Ministry of Education, Culture, Sports, Science and Technology (MEXT), Japan (2015) Standard tables of food composition in Japan - 2015 - (seventh revised version). https://www.mext.go. jp/en/policy/science_technology/policy/title01/detail01/1374030. htm. Accessed 7 June 2021

Nimura N, Kinoshita T (1980) Fluorescent labeling of fatty acids with 9-anthryldiazomethane (ADAM) for high performance liquid chromatography. Anal Lett 13:191-202

Noda H (1993) Health benefits and nutritional properties of nori. J Appl Phycol 5:255-258

Noda H, Amano H, Abo K, Horiguchi Y (1981) Sugars, organic acids, and minerals of 'nori' the dried laver Porphyra spp. Nippon Suisan Gakkaishi 47:57-62

Ohnishi K, Yoshida Y, Sekiguchi J (1994) Lipase production of Aspergillus oryzae. J Ferment Bioeng 77:490-495
Painter TJ (1983) Algal polysaccharides. In: Aspinall GO (ed) The polysaccharides. Academic Press, New York, pp 195-285

Rees DA, Conway E (1962) The structure and biosynthesis of porphyran: A comparison of some samples. Biochem J 84:411-416

Serhan CN, Chiang N, Van Dyke TE (2008) Resolving inflammation: dual anti-inflammatory and pro-resolution lipid mediators. Nat Rev Immunol 8:349-361

Suzuki T (1994) High-performance liquid chromatographic resolution of dinophysistoxin-1 and free fatty acids as 9-anthrylmethyl esters. J Chromatogr A 677:301-306

Swanson D, Block R, Mousa SA (2012) Omega-3 fatty acids EPA and DHA: health benefits throughout life. Adv Nutr 3:1-7

Uchida M, Kurushima H, Ishihara K, Murata Y, Touhata K, Ishida N, Niwa K, Araki T (2017) Characterization of fermented seaweed sauce prepared from nori (Pyropia yezoensis). J Biosci Bioeng 123:327-332

Uchida M, Kurushima H, Ishida N, Araki T, Ishihara K, Murata Y, Touhata K, Ishida N (2018) Preparation and characterization of fermented seaweed sauce manufactured from low-quality nori (dried and fresh fronds of Pyropia yezoensis). Fish Sci 84:589-596

Wells ML, Potin P, Craigie SJ, Raven JA, Merchant SS, Katherine E, Helliwell KE, Smith AG, Camire ME, Brawley SH (2017) Algae as nutritional and functional food sources: revisiting our understanding. J App Phycol 2:949-982

Yalpani M (1988) Structures. Polysaccharides: synthesis, modifications and structure/property relations. Elsevier, Amsterdam, pp 8-49

Yaphe W, Arsenault GP (1965) Improved resorcinol reagent for the determination of fructose, and of 3,6-anhydrogalactose in polysaccharides. Anal Biochem 13:143-148

Zhu Y, Tramper J (2013) Koji - where East meets West in fermentation. Biotechnol Adv 31:1448-1457

Publisher's note Springer Nature remains neutral with regard to jurisdictional claims in published maps and institutional affiliations. 